\title{
GENERALIZED FUZZY EULER-LAGRANGE EQUATIONS AND TRANSVERSALITY CONDITIONS
}

\author{
O.S. FARD, R. ALMEIDA, J. SOOLAKI, A.H. BORZABADI
}

\begin{abstract}
The study of fuzzy fractional variational problems in terms of a fractional Liouville-Caputo derivative is introduced. Necessary optimality conditions for problems of the fuzzy fractional calculus of variations with free end-points are proved, as well as transversality conditions.
\end{abstract}

\section{INTRODUCTION}

Fractional calculus is one of the generalizations of classical calculus and it has been used successfully in various fields of science and engineering. The real phenomena, such as dielectric and electrode-electrolyte polarization, electromagnetic waves, earthquakes, fluid dynamics, traffic, viscoelasticity and viscoplasticity, can be described successfully and more accurately using fractional models (see Kilbas et al. [19, Podlubny [25]).

Fractional variational calculus is one of the areas where considerable progress has been made. Riewe [26] was the first to initiate this field. Afterward, Agrawal [1] combined calculus of variations with fractional derivatives to develop EulerLagrange equations for fractional variational problems. The generalized EulerLagrange equation and transversality conditions for fractional variational problems, in terms of the Riemann-Liouville and the Liouville-Caputo derivatives, were presented in 2, 3] respectively. The necessary and sufficient optimality conditions for the fractional calculus of variations problems were also derived in [5, 20, 21, 22, The books written by Malinowska et al. 23] and Almeida et.al [6, and also Odzijewicz et al. 24, give many useful techniques for solving the fractional variational problems.

The fuzzy calculus of variations extends the classical variational calculus considering variables and their derivatives in fuzzy form. Although the notion of fuzzy set is widely spread to various problems such as optimization, differential equations and even fractional differential equations, very few works has been done to the fuzzy variational problems [12, 13, 15]. Recently, Farhadinia [15] studied necessary optimality conditions for fuzzy variational problems using the fuzzy differentiability concept due to Buckley and Feuring [9]. Farhadinia's work was generalized by Fard et al. [12, 13. In 12 Fard and Zadeh, using a $\alpha$-differentiability concept, obtained an extended fuzzy Euler-Lagrange condition. Fard et al. 13 presented the fuzzy Euler-Lagrange condition for fuzzy constrained and unconstrained variational problems under the generalized Hukuhara differentiability.

2010 Mathematics Subject Classification. 93C42 (Primary); 26A33, 34A07, 34A08, 93D05 (Secondary).

Key words and phrases. Fuzzy fractional variational problems, Fuzzy fractional EulerLagrange conditions, Fuzzy Liouville-Caputo derivative. 
Allahviranloo et al. in [4, 27] have studied the concepts about generalized Hukuhara fractional Riemann-Liouville and Liouville-Caputo differentiability of fuzzy-valued functions. Fard et al.14] investigated the fuzzy fractional EulerLagrange equation for the following functional

$$
\tilde{J}(\tilde{y})=\int_{a}^{b} \tilde{L}\left(x, \tilde{y}(x){ }_{a}^{C} D_{x}^{\alpha} \tilde{y}(x){ }_{x}^{C} D_{b}^{\beta} \tilde{y}(x)\right) d x .
$$

They considered the simple case where the lower bound (or upper bound) of $\tilde{J}$ is stated in terms containing only $\underline{y}^{r}(x){ }_{a}^{C} D_{x}^{\alpha} \underline{y}^{r}(x)$, and ${ }_{x}^{C} D_{b}^{\beta} \underline{y}^{r}(x)$ (or $\bar{y}^{r}(x)$, ${ }_{a}^{C} D_{x}^{\alpha} \bar{y}^{r}(x)$, and $\left.{ }_{x}^{C} D_{b}^{\beta} \bar{y}^{r}(x)\right)$. In this article the fuzzy Euler-Lagrange condition in 14 is generalized, such that both lower bound and upper bound of the functional are considered to be in terms containing $\underline{y}^{r}(x){ }_{a}^{C} D_{x}^{\alpha} \underline{y}^{r}(x){ }_{x}^{C} D_{b}^{\beta} \underline{y}^{r}(x), \bar{y}^{r}(x)$, ${ }_{a}^{C} D_{x}^{\alpha} \bar{y}^{r}(x)$ and ${ }_{x}^{C} D_{b}^{\beta} \bar{y}^{r}(x)$. Moreover, we develop the theory further by proving the necessary optimality conditions for more general problems of the fuzzy fractional calculus of variations with a Lagrangian that may also depend on the unspecified end-points. We also find the transversality conditions for when an end point lies on a given arbitrary curve.

The paper is organized as follows. Section 2 presents some notations on the fuzzy numbers space, differentiability and integrability of a fuzzy mapping. The main results concerning the fuzzy Euler-Lagrange equation for the FFVPs are established in Section 3. Section 4 presents the transversality condition for when an end point lies on a given arbitrary curve, and finally, conclusions are discussed in Section 5 .

\section{Preliminaries}

Let us denote by $\mathbb{R}_{f}$ the class of fuzzy numbers, i.e., normal, convex, upper semicontinuous and compactly supported fuzzy subsets of the real numbers. For $0<r \leq 1$, let $[\tilde{u}]^{r}=\{x \in \mathbb{R} \mid \tilde{u}(x) \geq r\}$ and $[\tilde{u}]^{0}=\overline{\{x \in \mathbb{R} \mid \tilde{u}(x) \geq 0\}}$. Then, it is well known that $[\tilde{u}]^{r}$ is a bounded closed interval for any $r \in[0,1]$.

Lemma 2.1 (See Theorem 1.1 of [16] and Lemma 2.1 of [29]). If $\underline{a}^{r}:[0,1] \rightarrow \mathbb{R}$ and $\bar{a}^{r}:[0,1] \rightarrow \mathbb{R}$ satisfy the conditions

(i) $\underline{a}^{r}:[0,1] \rightarrow \mathbb{R}$ is a bounded nondecreasing function,

(ii) $\bar{a}^{r}:[0,1] \rightarrow \mathbb{R}$ is a bounded nonincreasing function,

(iii) $\underline{a}^{1} \leq \bar{a}^{1}$,

(iv) for $0<k \leq 1, \lim _{r \rightarrow k^{-}} \underline{a}^{r}=\underline{a}^{k}$ and $\lim _{r \rightarrow k^{-}} \bar{a}^{r}=\bar{a}^{k}$,

(v) $\lim _{r \rightarrow 0^{+}} \underline{a}^{r}=\underline{a}^{0}$ and $\lim _{r \rightarrow 0^{+}} \bar{a}^{r}=\bar{a}^{0}$,

then $\tilde{a}: \mathbb{R} \rightarrow[0,1]$, characterized by $\tilde{a}(t)=\sup \left\{r \mid \underline{a}^{r} \leq t \leq \bar{a}^{r}\right\}$, is a fuzzy number with $[\tilde{a}]^{r}=\left[\underline{a}^{r}, \bar{a}^{r}\right]$. The converse is also true: if $\tilde{a}(t)=\sup \left\{r \mid \underline{a}^{r} \leq t \leq \bar{a}^{r}\right\}$ is a fuzzy number with parametrization given by $[\tilde{a}]^{r}=\left[\underline{a}^{r}, \bar{a}^{r}\right]$, then functions $\underline{a}^{r}$ and $\bar{a}^{r}$ satisfy conditions (i)-(v).

For $\tilde{u}, \tilde{v} \in \mathbb{R}_{f}$ and $\lambda \in \mathbb{R}$, the sum $\tilde{u}+\tilde{v}$ and the product $\lambda \cdot \tilde{u}$ are defined by $[\tilde{u}+\tilde{v}]^{r}=[\tilde{u}]^{r}+[\tilde{v}]^{r}$ and $[\lambda \cdot \tilde{u}]^{r}=\lambda[\tilde{u}]^{r}$ for all $r \in[0,1]$, where $[\tilde{u}]^{r}+[\tilde{v}]^{r}$ means the usual addition of two intervals (subsets) of $\mathbb{R}$ and $\lambda[\tilde{u}]^{r}$ means the usual product between a scalar and a subset of $\mathbb{R}$. The product $\tilde{u} \odot \tilde{v}$ of fuzzy numbers $\tilde{u}$ and $\tilde{v}$, is defined by $[\tilde{u} \odot \tilde{v}]^{r}=\left[\min \left\{\underline{u}^{r} \underline{v}^{r}, \underline{u}^{r} \bar{v}^{r}, \bar{u}^{r} \underline{v}^{r}, \bar{u}^{r} \bar{v}^{r}\right\}, \max \left\{\underline{u}^{r} \underline{v}^{r}, \underline{u}^{r} \bar{v}^{r}, \bar{u}^{r} \underline{v}^{r}, \bar{u}^{r} \bar{v}^{r}\right\}\right]$. 
The metric structure is given by the Hausdorff distance $D: \mathbb{R}_{f} \times \mathbb{R}_{f} \rightarrow \mathbb{R}_{+} \cup\{0\}$, $D(\tilde{u}, \tilde{v})=\sup _{r \in[0,1]} \max \left\{\left|\underline{u}^{r}-\underline{v}^{r}\right|,\left|\bar{u}^{r}-\bar{v}^{r}\right|\right\}$.

We say that the fuzzy number $\tilde{u}$ is triangular if $\underline{u}^{1}=\bar{u}^{1}, \underline{u}^{r}=\underline{u}^{1}-(1-r)\left(\underline{u}^{1}-\underline{u}^{0}\right)$ and $\bar{u}^{r}=\underline{u}^{1}-(1-r)\left(\bar{u}^{0}-\underline{u}^{1}\right)$. The triangular fuzzy number $u$ is generally denoted by $\tilde{u}=<\underline{u}^{0}, \underline{u}^{1}, \bar{u}^{0}>$. We define the fuzzy zero $\tilde{0}_{x}$ as

$$
\tilde{0}_{x}= \begin{cases}1 & \text { if } x=0 \\ 0 & \text { if } x \neq 0\end{cases}
$$

Definition 2.2 (See [15]). We say that $\tilde{f}:[a, b] \rightarrow \mathbb{R}_{f}$ is continuous at $x \in[a, b]$, if both $\underline{f}^{r}(x)$ and $\bar{f}^{r}(x)$ are continuous functions of $x \in[a, b]$ for all $r \in[0,1]$.

Definition 2.3 (See $[8$ ). The generalized Hukuhara difference of two fuzzy numbers $\tilde{x}, \tilde{y} \in \mathbb{R}_{f}$ ( $g H$-difference for short) is defined as follows:

$$
\tilde{x} \ominus_{g H} \tilde{y}=\tilde{z} \Leftrightarrow \tilde{x}=\tilde{y}+\tilde{z} \text { or } \tilde{y}=\tilde{x}+(-1) \tilde{z} .
$$

If $\tilde{z}=\tilde{x} \ominus_{g H} \tilde{y}$ exists as a fuzzy number, then its level cuts $\left[\underline{z}^{r}, \bar{z}^{r}\right]$ are obtained by $\underline{z}^{r}=\min \left\{\underline{x}^{r}-\underline{y}^{r}, \bar{x}^{r}-\bar{y}^{r}\right\}$ and $\bar{z}^{r}=\max \left\{\underline{x}^{r}-\underline{y}^{r}, \bar{x}^{r}-\bar{y}^{r}\right\}$ for all $r \in[0,1]$.

If the fuzzy function $\tilde{f}(x)$ is continuous in the metric $D$, then its definite integral exists. Furthermore,

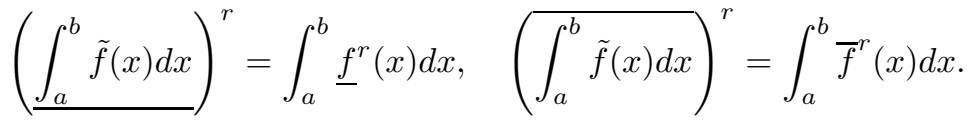

Definition 2.4 (See [15]). Let $\tilde{a}, \tilde{b} \in \mathbb{R}_{f}$. We write $\tilde{a} \preceq \tilde{b}$, if $\underline{a}^{r} \leq \underline{b}^{r}$ and $\bar{a}^{r} \leq \bar{b}^{r}$ for all $r \in[0,1]$. We also write $\tilde{a} \prec \tilde{b}$, if $\tilde{a} \preceq \tilde{b}$ and there exists an $r^{\prime} \in[0,1]$ so that $\underline{a}^{r^{\prime}}<\underline{b}^{r^{\prime}}$ and $\bar{a}^{r^{\prime}}<\bar{b}^{r^{\prime}}$. Moreover, $\tilde{a} \approx \tilde{b}$ if $\tilde{a} \preceq \tilde{b}$ and $\tilde{a} \succeq \tilde{b}$, that is, $[\tilde{a}]^{r}=[\tilde{b}]^{r}$ for all $r \in[0,1]$.

We say that $\tilde{a}, \tilde{b} \in \mathbb{R}_{f}$ are comparable if either $\tilde{a} \preceq \tilde{b}$ or $\tilde{a} \succeq \tilde{b}$; and noncomparable otherwise.

Definition 2.5. Let $T$ is an open subset of $\mathbb{R}$. A point $x_{0} \in T$ is a locally minimum (resp. maximum) of $\tilde{f}(x)$ if there exists some $\epsilon>0$ such that $\tilde{f}\left(x_{0}\right) \preceq \tilde{f}(x)$ (resp. $\left.\tilde{f}\left(x_{0}\right) \succeq \tilde{f}(x)\right)$ when $x \in N_{\epsilon}\left(x_{0}\right)$.

Theorem 2.6 (See 28). Let $f$ be a real-valued function differentiable on the open interval I. If $f$ has a local extremum at $x \in I$, then $\frac{d f}{d x}(x)=0$.

Lemma 2.7. If $x^{*}$ is a locally minimum of $\tilde{f}(x)$, then $x^{*}$ is also a locally minimum of the real-valued functions $\underline{f}^{r}(x)$ and $\bar{f}^{r}(x)$ for all $r \in[0,1]$. So we have $\frac{d \underline{f}^{r}}{d x}\left(x^{*}\right)=$ $\frac{d \bar{f}^{r}}{d x}\left(x^{*}\right)=0$.

Proof. In the neighborhood $N_{\epsilon}\left(x^{*}\right)$ we have $\tilde{f}\left(x^{*}\right) \preceq \tilde{f}(x)$. Using Definition 2.4 we get

$$
\underline{f}^{r}\left(x^{*}\right) \leq \underline{f}^{r}(x), \bar{f}^{r}\left(x^{*}\right) \leq \bar{f}^{r}(x)
$$

for all $r \in[0,1]$. So $x^{*}$ is also a local minimum of the real-valued functions $f^{r}(x)$ and $\bar{f}^{r}(x)$ for all $r \in[0,1]$ and by Theorem 2.6 we arrive at $\frac{d \underline{f}^{r}}{d x}\left(x^{*}\right)=\frac{d \bar{f}^{r}}{d x}\left(x^{*}\right)=0$. 
Definition 2.8 (See [15]). A fuzzy neighborhood $\tilde{N}_{\epsilon}(\tilde{\hat{f}})$ is the set of all curves $\tilde{f}$ satisfying for all $x \in\left[x_{0}, x_{f}\right]$

$$
D(\tilde{\hat{f}}(x), \tilde{f}(x))=\sup _{r \in[0,1]} \max \left\{\left|\underline{\hat{f}}^{r}(x)-\underline{f}^{r}(x)\right|,\left|\overline{\hat{f}}^{r}(x)-\bar{f}^{r}(x)\right|\right\} \leq \epsilon
$$

where $\epsilon$ is a small real number.

Definition 2.9. Let $\tilde{f}$ be a fuzzy function defined on the $\mathbb{R}_{f}$. The function $\tilde{f}$ is said to have a local minimum at $\tilde{\hat{x}}$ if there exists a number $\epsilon>0$ such that for any $\tilde{x} \in \tilde{N}_{\epsilon}(\tilde{\hat{x}}), \tilde{f}(\tilde{\hat{x}}) \preceq \tilde{f}(\tilde{x})$.

Definition 2.10. We say that the fuzzy curve $\tilde{f}(x)$ intersects the fuzzy curve $\tilde{g}(x)$ at $x_{0}$ if $\underline{f}^{1}\left(x_{0}\right)=\underline{g}^{1}\left(x_{0}\right)$ and $\bar{f}^{1}\left(x_{0}\right)=\bar{g}^{1}\left(x_{0}\right)$.

\section{Optimality for Fuzzy Fractional Variational Problems}

Following [27, we denote the space of all continuous fuzzy valued functions on $[a, b] \in \mathbb{R}$ by $C^{F}[a, b] ;$ the class of fuzzy functions with continuous first derivatives on $[a, b] \in \mathbb{R}$ by $C^{F 1}[a, b]$; and the space of all Lebesgue integrable fuzzy valued functions on the bounded interval $[a, b]$ is indicated by $L^{F}[a, b]$.

Let us consider the following problem:

$$
\begin{gathered}
\tilde{J}(\tilde{y})=\int_{a}^{b} \tilde{L}\left(x, \tilde{y}(x),{ }^{g H-C}{ }_{a} \mathcal{D}_{i x}^{\alpha} \tilde{y}(x),{ }^{g H-C}{ }_{x} \mathcal{D}_{i b}^{\beta} \tilde{y}(x),\right. \\
\tilde{y}(a), \tilde{y}(b)) d x \longrightarrow \text { extr, } \quad \mathrm{i}=1,2, \\
\left(\tilde{y}(a)=\tilde{y}_{a}\right), \quad\left(\tilde{y}(b)=\tilde{y}_{b}\right) .
\end{gathered}
$$

Here, the Lagrange function $\tilde{L}$ is assumed to be of class $C^{F 1}$ on all its arguments. To fix notation, we consider $\alpha, \beta \in(0,1)$. Here ${ }^{g H-C_{a}} \mathcal{D}_{i x}^{\alpha} \tilde{y}(x)$ and ${ }^{g H-C}{ }_{x} \mathcal{D}_{i b}^{\beta} \tilde{y}(x)$ denote the left Liouville-Caputo Fuzzy Fractional Derivative (LCFFD) and the right Liouville-Caputo Fuzzy Fractional Derivative (RCFFD), respectively:

for $i=1,2$.

$$
\begin{aligned}
{ }^{g H-C}{ }_{a} \mathcal{D}_{i x}^{\alpha} \tilde{y}(x) & =\frac{1}{\Gamma(1-\alpha)} \int_{a}^{x}(x-t)^{-\alpha}\left(\mathcal{D}_{i, g H} \tilde{y}\right)(t) d t, \\
{ }_{g H-C}{ }_{x} \mathcal{D}_{i b}^{\beta} \tilde{y}(x) & =\frac{-1}{\Gamma(1-\beta)} \int_{x}^{b}(t-x)^{-\beta}\left(\mathcal{D}_{i, g H} \tilde{y}\right)(t) d t
\end{aligned}
$$

Remark 3.1. We use the notation ${ }^{g H-C}{ }_{a} \mathcal{D}_{i x}^{\beta} \tilde{f}$ when the fuzzy-valued function $\tilde{f}$ is $[(i)-g H]_{\alpha}^{C}$-differentiable with respect to the independent variable $x, i \in\{1,2\}$.

Definition 3.2 (See [4]). Let $\alpha \in[0,1]$ and $\tilde{f}:[a, b] \rightarrow \mathbb{R}_{f}$ be $[g H]_{\alpha}^{C}$-differentiable at $x \in[a, b]$. We say that $\tilde{f}$ is $[(1)-g H]_{\alpha}^{C}$-differentiable at $x \in[a, b]$ if

$$
\left[{ }_{a}^{g H-C} \mathcal{D}_{x}^{\alpha} \tilde{f}(x)\right]^{r}=\left[{ }_{a}^{C} D_{x}^{\alpha} \underline{f}^{r}(x),{ }_{a}^{C} D_{x}^{\alpha} \bar{f}^{r}(x)\right], \quad 0 \leq r \leq 1,
$$

and that $\tilde{f}$ is $[(2)-g H]_{\alpha}^{C}$-differentiable at $x$ if

$$
\left[{ }_{a}^{g H-C} \mathcal{D}_{x}^{\alpha} \tilde{f}(x)\right]^{r}=\left[{ }_{a}^{C} D_{x}^{\alpha} \bar{f}^{r}(x),{ }_{a}^{C} D_{x}^{\alpha} \underline{f}^{r}(x)\right], \quad 0 \leq r \leq 1 .
$$

Remark 3.3. We use the notation ${ }^{g H-C}{ }_{a} \mathcal{D}_{i x}^{\beta} \tilde{f}$ when the fuzzy-valued function $\tilde{f}$ is $[(i)-g H]_{\alpha}^{C}$-differentiable with respect to the independent variable $x, i \in\{1,2\}$. 
Along the work we denote by $\partial_{i} L$ the partial derivative of function $L$ with respect to its $i$ th argument. To develop the necessary conditions for the extremum for (2), assume that $\tilde{y}^{*}(x)$ is the desired function, let $\epsilon \in \mathbb{R}$, and define a family of curves $\tilde{y}(x)=\tilde{y}^{*}(x)+\epsilon \tilde{h}(x)$, where $\tilde{h}$ is an arbitrary admissible variation. We do not require $\tilde{h}(a)=\tilde{0}$ or $\tilde{h}(b)=\tilde{0}$ in the case when $\tilde{y}(a)$ or $\tilde{y}(b)$, respectively, is free (it is possible that both are free). Let

$$
\begin{gathered}
\tilde{J}(\epsilon)=\int_{a}^{b} \tilde{L}\left(x, \tilde{y}^{*}(x)+\epsilon \tilde{h}(x),{ }^{g H-C}{ }_{a} \mathcal{D}_{i x}^{\alpha}\left(\tilde{y}^{*}+\epsilon \tilde{h}(x)\right),{ }^{g H-C}{ }_{x} \mathcal{D}_{i b}^{\beta}\left(\tilde{y}^{*}+\epsilon \tilde{h}(x)\right),\right. \\
\left.\tilde{y}^{*}(a)+\epsilon \tilde{h}(a), \tilde{y}^{*}(b)+\epsilon \tilde{h}(b)\right) d x
\end{gathered}
$$

for $i=1,2$. The lower bound and upper bound of $\tilde{J}$ are

$$
\underline{J}^{r}(\epsilon)=\int_{a}^{b}\left\{\underline{L}^{r}\left[x, \tilde{y}^{*}(x)+\epsilon \tilde{h}(x), \tilde{y}^{*}(a)+\epsilon \tilde{h}(a), \tilde{y}^{*}(b)+\epsilon \tilde{h}(b)\right]^{r}\right\} d x
$$

and

$$
\bar{J}^{r}(\epsilon)=\int_{a}^{b}\left\{\bar{L}^{r}\left[x, \tilde{y}^{*}(x)+\epsilon \tilde{h}(x), \tilde{y}^{*}(a)+\epsilon \tilde{h}(a), \tilde{y}^{*}(b)+\epsilon \tilde{h}(b)\right]^{r}\right\} d x .
$$

respectively, where

$$
\begin{aligned}
{\left[x, \tilde{y}^{*}(x)+\epsilon \tilde{h}(x), \tilde{y}^{*}(a)+\epsilon \tilde{h}(a), \tilde{y}^{*}(b)+\epsilon \tilde{h}(b)\right]^{r} } & \\
=(x, & \underline{y}^{* r}(x)+\epsilon \underline{h}^{r}(x), \bar{y}^{* r}(x)+\epsilon \bar{h}^{r}(x),{ }_{a}^{C} D_{x}^{\alpha}\left(\underline{y}^{* r}+\epsilon \underline{h}^{r}(x)\right), \\
& { }_{a}^{C} D_{x}^{\alpha}\left(\bar{y}^{* r}+\epsilon \bar{h}^{r}(x)\right),{ }_{x}^{C} D_{b}^{\beta}\left(\underline{y}^{* r}+\epsilon \underline{h}^{r}(x)\right),{ }_{x}^{C} D_{b}^{\beta}\left(\bar{y}^{* r}+\epsilon \bar{h}^{r}(x)\right), \\
& \left.\underline{y}^{r}(a)+\epsilon \underline{h}^{r}(a), \bar{y}^{* r}(a)+\epsilon \bar{h}^{r}(a), \underline{y}^{* r}(b)+\epsilon \underline{h}^{r}(b), \bar{y}^{* r}(b)+\epsilon \bar{h}^{r}(b)\right) .
\end{aligned}
$$

By Lemma 2.7. $\tilde{J}(\epsilon)$ is extremum at $\epsilon=0$, therefore necessary conditions for $\tilde{y}$ to be an extremizer are given by setting $\frac{d J^{r}}{d \epsilon}=0, \frac{d \bar{J}^{r}}{d \epsilon}=0$, at $\epsilon=0$, for all admissible $\tilde{h}(x)$. Differentiating $\underline{J}^{r}$, we obtain

$$
\begin{aligned}
\left.\frac{d J^{r}}{d \epsilon}\right|_{\epsilon=0} & =0 \longrightarrow \\
\int_{a}^{b} & {\left[\partial_{2} \underline{L}^{r}(\ldots) \underline{h}^{r}+\partial_{3} \underline{L}^{r}(\ldots) \bar{h}^{r}+\partial_{4} \underline{L}^{r}(\ldots)_{a}^{C} D_{x}^{\alpha} \underline{h}^{r}(x)+\partial_{5} \underline{L}^{r}(\ldots)_{a}^{C} D_{x}^{\alpha} \bar{h}^{r}(x)\right.} \\
& +\partial_{6} \underline{L}(\ldots)_{x}^{C} D_{b}^{\beta} \underline{h}^{r}(x)+\partial_{7} \underline{L}^{r}(\ldots)_{x}^{C} D_{b}^{\beta} \bar{h}^{r}(x)+\partial_{8} \underline{L}^{r}(\ldots) \underline{h}^{r}(a) \\
& \left.+\partial_{9} \underline{L}^{r}(\ldots) \bar{h}^{r}(a)+\partial_{10} \underline{L}(\ldots) \underline{h}^{r}(b)+\partial_{11} \underline{L}^{r}(\ldots) \bar{h}^{r}(b)\right] d x=0
\end{aligned}
$$

where

$$
\begin{aligned}
(\ldots)=\left(x, \underline{y}^{* r}(x), \bar{y}^{* r}(x),{ }_{a}^{C} D_{x}^{\alpha} \underline{y}^{* r},{ }_{a}^{C} D_{x}^{\alpha} \bar{y}^{* r},{ }_{x}^{C} D_{b}^{\beta} \underline{y}^{* r},\right. \\
\left.{ }_{x}^{C} D_{b}^{\beta} \bar{y}^{* r}, \underline{y}^{* r}(a), \bar{y}^{* r}(a), \underline{y}^{* r}(b), \bar{y}^{* r}(b)\right) .
\end{aligned}
$$


We consider Eq. (3). Using integration by parts,

$$
\begin{aligned}
& \int_{a}^{b} \partial_{4} \underline{L}^{r}(\ldots)_{a}^{C} D_{x}^{\alpha} \underline{h}^{r}(x) d x=\int_{a}^{b} \underline{h}^{r}(x)_{x} D_{b}^{\alpha} \partial_{4} \underline{L}^{r}(\ldots) d x+\left.{ }_{x} I_{b}^{1-\alpha} \partial_{4} \underline{L}^{r}(\ldots) \underline{h}^{r}(x)\right|_{x=a} ^{x=b}, \\
& \int_{a}^{b} \partial_{5} \underline{L}^{r}(\ldots)_{a}^{C} D_{x}^{\alpha} \bar{h}^{r}(x) d x=\int_{a}^{b} \bar{h}^{r}(x)_{x} D_{b}^{\alpha} \partial_{5} \underline{L}^{r}(\ldots) d x+\left.{ }_{x} I_{b}^{1-\alpha} \partial_{5} \underline{L}^{r}(\ldots) \bar{h}^{r}(x)\right|_{x=a} ^{x=b}, \\
& \int_{a}^{b} \partial_{6} \underline{L}^{r}(\ldots)_{x}^{C} D_{b}^{\beta} \underline{h}^{r}(x) d x=\int_{a}^{b} \underline{h}^{r}(x)_{a} D_{x}^{\beta} \partial_{6} \underline{L}^{r}(\ldots) d x-\left.{ }_{a} I_{x}^{1-\beta} \partial_{6} \underline{L}^{r}(\ldots) \underline{h}^{r}(x)\right|_{x=a} ^{x=b}, \\
& \int_{a}^{b} \partial_{7} \underline{L}^{r}(\ldots)_{x}^{C} D_{b}^{\beta} \bar{h}^{r}(x) d x=\int_{a}^{b} \bar{h}^{r}(x)_{a} D_{x}^{\beta} \partial_{7} \underline{L}^{r}(\ldots) d x-\left.{ }_{a} I_{x}^{1-\beta} \partial_{7} \underline{L}^{r}(\ldots) \bar{h}^{r}(x)\right|_{x=a} ^{x=b} .
\end{aligned}
$$

Substituting Eqs. (4) into Eq. (3), we get

$$
\begin{aligned}
& \int_{a}^{b}\left\{\left[\partial_{2} \underline{L}^{r}(\ldots)+{ }_{x} D_{b}^{\alpha} \partial_{4} \underline{L}^{r}(\ldots)+{ }_{a} D_{x}^{\beta} \partial_{6} \underline{L}^{r}(\ldots)\right] \underline{h}^{r}(x)+\left[\partial_{3} \underline{L}^{r}(\ldots)+{ }_{x} D_{b}^{\alpha} \partial_{5} \underline{L}^{r}(\ldots)\right.\right. \\
& \left.\left.\quad+{ }_{a} D_{x}^{\beta} \partial_{7} \underline{L}^{r}(\ldots)\right] \bar{h}^{r}(x)\right\} d x+\left.\left({ }_{x} I_{b}^{1-\alpha} \partial_{4} \underline{L}^{r}(\ldots)-{ }_{a} I_{x}^{1-\beta} \partial_{6} \underline{L}^{r}(\ldots)\right) \underline{h}^{r}(x)\right|_{x=a} ^{x=b} \\
& \quad+\left.\left({ }_{x} I_{b}^{1-\alpha} \partial_{5} \underline{L}^{r}(\ldots)-{ }_{a} I_{x}^{1-\beta} \partial_{7} \underline{L}^{r}(\ldots)\right) \bar{h}^{r}(x)\right|_{x=a} ^{x=b}+\int_{a}^{b}\left\{\partial_{8} \underline{L}^{r}(\ldots) \underline{h}^{r}(a)\right. \\
& \left.\quad+\partial_{9} \underline{L}^{r}(\ldots) \bar{h}^{r}(a)+\partial_{10} \underline{L}^{r}(\ldots) \underline{h}^{r}(b)+\partial_{11} \underline{L}^{r}(\ldots) \bar{h}^{r}(b)\right\} d x=0
\end{aligned}
$$

We first consider functions $\underline{h}^{r}$ and $\bar{h}^{r}$ such that $\underline{h}^{r}(a)=\bar{h}^{r}(a)=\underline{h}^{r}(b)=\bar{h}^{r}(b)=0$. Then, by the fundamental lemma of the calculus of variations, we deduce that

$$
\begin{aligned}
& \partial_{2} \underline{L}^{r}(\ldots)+{ }_{x} D_{b}^{\alpha} \partial_{4} \underline{L}^{r}(\ldots)+{ }_{a} D_{x}^{\beta} \partial_{6} \underline{L}^{r}(\ldots)=0, \\
& \partial_{3} \underline{L}^{r}(\ldots)+{ }_{x} D_{b}^{\alpha} \partial_{5} \underline{L}^{r}(\ldots)+{ }_{a} D_{x}^{\beta} \partial_{7} \underline{L}^{r}(\ldots)=0 .
\end{aligned}
$$

for all $x \in[a, b]$. Therefore, in order for $\tilde{y}^{*}$ to be an extremizer to the problem (2), $\tilde{y}^{*}$ must be a solution of the fuzzy fractional Euler-Lagrange equations (6) and (7). But if $\tilde{y}^{*}$ is a solution of (6) and (7), the first integral in expression (5) vanishes, then the condition (3) takes the form

$$
\begin{aligned}
& \underline{h}^{r}(a)\left[\int_{a}^{b} \partial_{8} \underline{L}^{r}(\ldots) d x-\left.\left({ }_{x} I_{b}^{1-\alpha} \partial_{4} \underline{L}^{r}(\ldots)-{ }_{a} I_{x}^{1-\beta} \partial_{6} \underline{L}^{r}(\ldots)\right)\right|_{x=a}\right] \\
& +\bar{h}^{r}(a)\left[\int_{a}^{b} \partial_{9} \underline{L}^{r}(\ldots) d x-\left.\left({ }_{x} I_{b}^{1-\alpha} \partial_{5} \underline{L}^{r}(\ldots)-{ }_{a} I_{x}^{1-\beta} \partial_{7} \underline{L}^{r}(\ldots)\right)\right|_{x=a}\right] \\
& +\underline{h}^{r}(b)\left[\int_{a}^{b} \partial_{10} \underline{L}^{r}(\ldots) d x+\left.\left({ }_{x} I_{b}^{1-\alpha} \partial_{4} \underline{L}^{r}(\ldots)-{ }_{a} I_{x}^{1-\beta} \partial_{6} \underline{L}^{r}(\ldots)\right)\right|_{x=b}\right] \\
& +\bar{h}^{r}(b)\left[\int_{a}^{b} \partial_{11} \underline{L}^{r}(\ldots) d x+\left.\left({ }_{x} I_{b}^{1-\alpha} \partial_{5} \underline{L}^{r}(\ldots)-{ }_{a} I_{x}^{1-\beta} \partial_{7} \underline{L}^{r}(\ldots)\right)\right|_{x=b}\right]=0 .
\end{aligned}
$$

If $\tilde{y}(a)=\tilde{y}_{a}$ and $\tilde{y}(b)=\tilde{y}_{b}$ are given in the formulation of problem (2), then the latter equation is trivially satisfied since $\tilde{h}(a)=\tilde{h}(b)=\tilde{0}$. Because $\underline{h}^{r}(a), \bar{h}^{r}(a)$ are arbitrary, when $\tilde{y}(a)$ is free, we have

$$
\int_{a}^{b} \partial_{8} \underline{L}^{r}(\ldots) d x-\left.\left({ }_{x} I_{b}^{1-\alpha} \partial_{4} \underline{L}^{r}(\ldots)-{ }_{a} I_{x}^{1-\beta} \partial_{6} \underline{L}^{r}(\ldots)\right)\right|_{x=a}=0,
$$




$$
\int_{a}^{b} \partial_{9} \underline{L}^{r}(\ldots) d x-\left.\left({ }_{x} I_{b}^{1-\alpha} \partial_{5} \underline{L}^{r}(\ldots)-{ }_{a} I_{x}^{1-\beta} \partial_{7} \underline{L}^{r}(\ldots)\right)\right|_{x=a}=0 .
$$

When $\tilde{y}(b)$ is free, we get

$$
\begin{aligned}
& \int_{a}^{b} \partial_{10} \underline{L}^{r}(\ldots) d x+\left.\left({ }_{x} I_{b}^{1-\alpha} \partial_{4} \underline{L}^{r}(\ldots)-{ }_{a} I_{x}^{1-\beta} \partial_{6} \underline{L}^{r}(\ldots)\right)\right|_{x=b}=0, \\
& \int_{a}^{b} \partial_{11} \underline{L}^{r}(\ldots) d x+\left.\left({ }_{x} I_{b}^{1-\alpha} \partial_{5} \underline{L}^{r}(\ldots)-{ }_{a} I_{x}^{1-\beta} \partial_{7} \underline{L}^{r}(\ldots)\right)\right|_{x=b}=0,
\end{aligned}
$$

because $\underline{h}^{r}(b)$ and $\bar{h}^{r}(b)$ are arbitrary. Following the scheme of obtaining (6)-(11) and adapting it to the case under consideration $\frac{d \bar{J}^{r}}{d \epsilon}=0$, one can show that

$$
\begin{aligned}
& \partial_{2} \bar{L}^{r}(\ldots)+{ }_{x} D_{b}^{\alpha} \partial_{4} \bar{L}^{r}(\ldots)+{ }_{a} D_{x}^{\beta} \partial_{6} \bar{L}^{r}(\ldots)=0, \\
& \partial_{3} \bar{L}^{r}(\ldots)+{ }_{x} D_{b}^{\alpha} \partial_{5} \bar{L}^{r}(\ldots)+{ }_{a} D_{x}^{\beta} \partial_{7} \bar{L}^{r}(\ldots)=0,
\end{aligned}
$$

for all $x \in[a, b]$. Moreover, if $\tilde{y}(a)$ is not specified, then

$$
\begin{aligned}
& \int_{a}^{b} \partial_{8} \bar{L}^{r}(\ldots) d x-\left.\left({ }_{x} I_{b}^{1-\alpha} \partial_{4} \bar{L}^{r}(\ldots)-{ }_{a} I_{x}^{1-\beta} \partial_{6} \bar{L}^{r}(\ldots)\right)\right|_{x=a}=0, \\
& \int_{a}^{b} \partial_{9} \bar{L}^{r}(\ldots) d x-\left.\left({ }_{x} I_{b}^{1-\alpha} \partial_{5} \bar{L}^{r}(\ldots)-{ }_{a} I_{x}^{1-\beta} \partial_{7} \bar{L}^{r}(\ldots)\right)\right|_{x=a}=0,
\end{aligned}
$$

and when $\tilde{y}(b)$ is free, then

$$
\begin{aligned}
& \int_{a}^{b} \partial_{10} \bar{L}^{r}(\ldots) d x+\left.\left({ }_{x} I_{b}^{1-\alpha} \partial_{4} \bar{L}^{r}(\ldots)-{ }_{a} I_{x}^{1-\beta} \partial_{6} \bar{L}^{r}(\ldots)\right)\right|_{x=b}=0 \\
& \int_{a}^{b} \partial_{11} \bar{L}^{r}(\ldots) d x+\left.\left({ }_{x} I_{b}^{1-\alpha} \partial_{5} \bar{L}^{r}(\ldots)-{ }_{a} I_{x}^{1-\beta} \partial_{7} \bar{L}^{r}(\ldots)\right)\right|_{x=b}=0 .
\end{aligned}
$$

Now we are in a position to state the necessary conditions for a relative (local) minimum (maximum) of problem (2), as follows:

Theorem 3.4. Let $\tilde{y}^{*}$ be a local extremizer to problem (2). Then, $\tilde{y}$ satisfies the fractional Euler-Lagrange equations

$$
\left\{\begin{array}{l}
\partial_{2} \underline{L}^{r}(\ldots)+{ }_{x} D_{b}^{\alpha} \partial_{4} \underline{L}^{r}(\ldots)+{ }_{a} D_{x}^{\beta} \partial_{6} \underline{L}^{r}(\ldots)=0, \\
\partial_{2} \bar{L}^{r}(\ldots)+{ }_{x} D_{b}^{\alpha} \partial_{4} \bar{L}^{r}(\ldots)+{ }_{a} D_{x}^{\beta} \partial_{6} \bar{L}^{r}(\ldots)=0, \\
\partial_{3} \underline{L}^{r}(\ldots)+{ }_{x} D_{b}^{\alpha} \partial_{5} \underline{L}^{r}(\ldots)+{ }_{a} D_{x}^{\beta} \partial_{7} \underline{L}^{r}(\ldots)=0, \\
\partial_{3} \bar{L}^{r}(\ldots)+{ }_{x} D_{b}^{\alpha} \partial_{5} \bar{L}^{r}(\ldots)+{ }_{a} D_{x}^{\beta} \partial_{7} \underline{L}^{r}(\ldots)=0 .
\end{array}\right.
$$

for all $x \in[a, b]$. Moreover, if $\tilde{y}(a)$ is free, then

$$
\left\{\begin{array}{l}
\int_{a}^{b} \partial_{8} \underline{L}^{r}(\ldots) d x-\left.\left({ }_{x} I_{b}^{1-\alpha} \partial_{4} \underline{L}^{r}(\ldots)-{ }_{a} I_{x}^{1-\beta} \partial_{6} \underline{L}^{r}(\ldots)\right)\right|_{x=a}=0 \\
\int_{a}^{b} \partial_{8} \bar{L}^{r}(\ldots) d x-\left.\left({ }_{x} I_{b}^{1-\alpha} \partial_{4} \bar{L}^{r}(\ldots)-{ }_{a} I_{x}^{1-\beta} \partial_{6} \bar{L}^{r}(\ldots)\right)\right|_{x=a}=0 \\
\int_{a}^{b} \partial_{9} \underline{L}^{r}(\ldots) d x-\left.\left({ }_{x} I_{b}^{1-\alpha} \partial_{5} \underline{L}^{r}(\ldots)-{ }_{a} I_{x}^{1-\beta} \partial_{7} \underline{L}^{r}(\ldots)\right)\right|_{x=a}=0 \\
\int_{a}^{b} \partial_{9} \bar{L}^{r}(\ldots) d x-\left.\left({ }_{x} I_{b}^{1-\alpha} \partial_{5} \bar{L}^{r}(\ldots)-{ }_{a} I_{x}^{1-\beta} \partial_{7} \bar{L}^{r}(\ldots)\right)\right|_{x=a}=0
\end{array}\right.
$$

and when $\tilde{y}(b)$ is free, then

$$
\left\{\begin{array}{l}
\int_{a}^{b} \partial_{10} \underline{L}^{r}(\ldots) d x+\left.\left({ }_{x} I_{b}^{1-\alpha} \partial_{4} \underline{L}^{r}(\ldots)-{ }_{a} I_{x}^{1-\beta} \partial_{6} \underline{L}^{r}(\ldots)\right)\right|_{x=b}=0 \\
\int_{a}^{b} \partial_{10} \bar{L}^{r}(\ldots) d x+\left.\left({ }_{x} I_{b}^{1-\alpha} \partial_{4} \bar{L}^{r}(\ldots)-{ }_{a} I_{x}^{1-\beta} \partial_{6} \bar{L}^{r}(\ldots)\right)\right|_{x=b}=0 \\
\int_{a}^{b} \partial_{11} \underline{L}^{r}(\ldots) d x+\left.\left({ }_{x} I_{b}^{1-\alpha} \partial_{5} \underline{L}^{r}(\ldots)-{ }_{a} I_{x}^{1-\beta} \partial_{7} \underline{L}^{r}(\ldots)\right)\right|_{x=b}=0 \\
\int_{a}^{b} \partial_{11} \bar{L}^{r}(\ldots) d x+\left.\left({ }_{x} I_{b}^{1-\alpha} \partial_{5} \bar{L}^{r}(\ldots)-{ }_{a} I_{x}^{1-\beta} \partial_{7} \bar{L}^{r}(\ldots)\right)\right|_{x=b}=0
\end{array}\right.
$$


where

$$
\begin{aligned}
(\ldots)=\left(x, \underline{y}^{* r}(x), \bar{y}^{* r}(x),{ }_{a}^{C} D_{x}^{\alpha} \underline{y}^{* r},{ }_{a}^{C} D_{x}^{\alpha} \bar{y}^{* r},{ }_{x}^{C} D_{b}^{\beta} \underline{y}^{* r},\right. \\
\left.{ }_{x}^{C} D_{b}^{\beta} \bar{y}^{* r}, \underline{y}^{* r}(a), \bar{y}^{* r}(a), \underline{y}^{* r}(b), \bar{y}^{* r}(b)\right) .
\end{aligned}
$$

Example 3.1. Let us consider the following problem:

$$
\tilde{J}(\tilde{y})=\frac{1}{2} \int_{0}^{1}\left({ }^{g H-C}{ }_{0} \mathcal{D}_{x}^{\alpha} \tilde{y}(x)\right)^{2}+\tilde{2} \odot \tilde{y}^{2}(0)+\tilde{3} \odot(\tilde{y}(1)-1)^{2} d x \longrightarrow \min ,
$$

where $\tilde{2}=<1,2,3>, \tilde{3}=<3,3,3>$.

Solution. We first derive the $r$-level set of $\tilde{J}$ for [(1)-gH]-differentiability of $\tilde{y}$ as follows:

$$
\begin{aligned}
{[\tilde{J}(\tilde{y})]^{r}=\left[\frac{1}{2} \int_{0}^{1}\left({ }_{0}^{C} D_{x}^{\alpha} \underline{y}^{r}(x)\right)^{2}+(3-r)\left(\underline{y}^{r}\right)^{2}(0)+3\left(\underline{y}^{r}(1)-1\right)^{2} d x,\right.} \\
\left.\frac{1}{2} \int_{0}^{1}\left({ }_{0}^{C} D_{x}^{\alpha} \bar{y}^{r}(x)\right)^{2}+(r+1)\left(\bar{y}^{r}\right)^{2}(0)+3\left(\bar{y}^{r}(1)-1\right)^{2} d x\right] .
\end{aligned}
$$

For this problem, the generalized Euler-Lagrange equations and the natural boundary conditions (see Theorem 3.4) are given as

$$
\begin{gathered}
{ }_{x} D_{1}^{\alpha}\left({ }_{0}^{C} D_{x}^{\alpha} \underline{y}^{r}(x)\right)=0, \\
{ }_{x} D_{1}^{\alpha}\left({ }_{0}^{C} D_{x}^{\alpha} \bar{y}^{r}(x)\right)=0, \\
\int_{0}^{1}(3-r) \underline{y}^{r}(0) d x=\left.{ }_{x} I_{1}^{1-\alpha}\left({ }_{0}^{C} D_{x}^{\alpha} \underline{y}^{r}(x)\right)\right|_{x=0}, \\
\int_{0}^{1} 3\left(\underline{y}^{r}(1)-1\right) d x=-\left.{ }_{x} I_{1}^{1-\alpha}\left({ }_{0}^{C} D_{x}^{\alpha} \underline{y}^{r}(x)\right)\right|_{x=1}, \\
\int_{0}^{1}(r+1) \bar{y}^{r}(0) d x=\left.{ }_{x} I_{1}^{1-\alpha}\left({ }_{0}^{C} D_{x}^{\alpha} \bar{y}^{r}(x)\right)\right|_{x=0}, \\
\left.\int_{0}^{1} 3\left(\bar{y}^{r}(1)-1\right) d x=-{ }_{x} I_{1}^{1-\alpha}{ }_{0}^{C} D_{x}^{\alpha} \bar{y}^{r}(x)\right)\left.\right|_{x=1} .
\end{gathered}
$$

Note that it is difficult to solve the above fractional equations. For $0<\alpha<1$, a numerical method should be used. When $\alpha$ goes to 1 , problem (12) tends to

$$
\tilde{J}(\tilde{y})=\frac{1}{2} \int_{0}^{1}\left(\tilde{y}^{\prime}(x)\right)^{2}+\tilde{2} \odot \tilde{y}^{2}(0)+\tilde{3} \odot(\tilde{y}(1)-1)^{2} d x \longrightarrow \min ,
$$

and Eqs. (13) could be replaced with

$$
\begin{gathered}
\left(\underline{y}^{r}\right)^{\prime \prime}(x)=0, \\
\left(\bar{y}^{r}\right)^{\prime \prime}(x)=0, \\
(3-r) \underline{y}^{r}(0)=\left(\underline{y}^{r}\right)^{\prime}(0), \\
3\left(\underline{y}^{r}(1)-1\right)=-\left(\underline{y}^{r}\right)^{\prime}(1), \\
(r+1) \bar{y}^{r}(0)=\left(\bar{y}^{r}\right)^{\prime}(0), \\
3\left(\bar{y}^{r}(1)-1\right)=-\left(\bar{y}^{r}\right)^{\prime}(1) .
\end{gathered}
$$




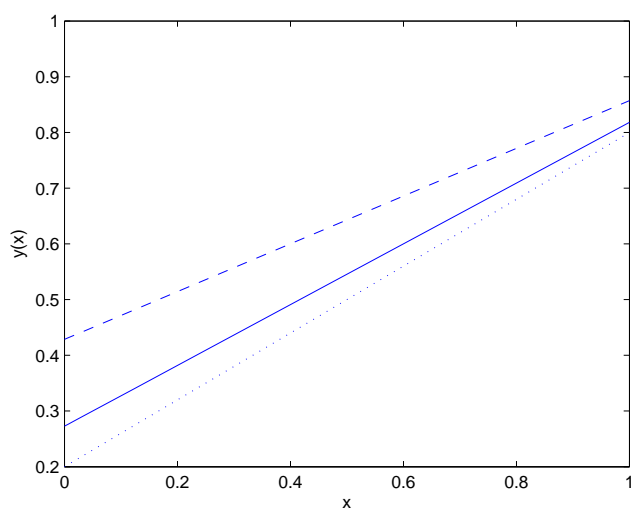

Figure 1. Solution of Example 3.1.

$\underline{y}^{0}(x)$ (dotted line), $\bar{y}^{0}(x)$ (dashed line), $\underline{y}^{1}(x)=\bar{y}^{1}(x)$ (solid line).

By solving Eqs. (15) we have

$$
\underline{y}^{r}(x)=\frac{-3 r+9}{-4 r+15} x+\frac{3}{-4 r+15}, \bar{y}^{r}(x)=\frac{3 r+3}{4 r+7} x+\frac{3}{4 r+7} .
$$

One can easily show that $\underline{y}^{r}(x)$ and $\bar{y}^{r}(x)$ satisfy Lemma2.1. This solution is shown in Figure 1

We now consider a more general case. We have assumed that the interval of integration of the functional (2) is the same of the admissible functions. We generalize Theorem 3.4 by considering a subinterval $[A, B] \subset[a, b]$ and the following optimization problem:

$$
\begin{gathered}
\tilde{J}(\tilde{y})=\int_{A}^{B} \tilde{L}\left(x, \tilde{y}(x),{ }^{g H-C}{ }_{a} \mathcal{D}_{i x}^{\alpha} \tilde{y}(x),{ }^{g H-C}{ }_{x} \mathcal{D}_{i b}^{\beta} \tilde{y}(x),\right. \\
\tilde{y}(a), \tilde{y}(A), \tilde{y}(B), \tilde{y}(b)) d x \longrightarrow \operatorname{extr}, \quad \mathrm{i}=1,2, \\
\left(\tilde{y}(a)=\tilde{y}_{a}\right), \quad\left(\tilde{y}(A)=\tilde{y}_{A}\right),\left(\tilde{y}(B)=\tilde{y}_{B}\right), \quad\left(\tilde{y}(b)=\tilde{y}_{b}\right) .
\end{gathered}
$$

Again, the function $\tilde{L}$ is assumed to be of class $C^{F 1}$ on all its arguments. In a similar way as done before, let $\tilde{y}^{*}(x)$ be an extremum for (16),,$\epsilon \in \mathbb{R}$ a real, and consider the variation functions $\tilde{y}(x)=\tilde{y}^{*}(x)+\epsilon \tilde{h}(x)$, where $\tilde{h}$ is an arbitrary admissible variation, which may satisfy or not the boundary conditions $\tilde{h}(a)=\tilde{0}, \tilde{h}(A)=\tilde{0}$, $\tilde{h}(B)=\tilde{0}$ or $\tilde{h}(b)=\tilde{0}$, depending if $\tilde{y}(a), \tilde{y}(A), \tilde{y}(B)$ or $\tilde{y}(b)$, is fixed or not. Define

$$
\begin{gathered}
\tilde{J}(\epsilon)=\int_{A}^{B} \tilde{L}\left(x, \tilde{y}^{*}(x)+\epsilon \tilde{h}(x),{ }^{g H-C}{ }_{a} \mathcal{D}_{i x}^{\alpha}\left(\tilde{y}^{*}+\epsilon \tilde{h}(x)\right),{ }^{g H-C}{ }_{x} \mathcal{D}_{i b}^{\alpha}\left(\tilde{y}^{*}+\epsilon \tilde{h}(x)\right),\right. \\
\left.\tilde{y}^{*}(a)+\epsilon \tilde{h}(a), \tilde{y}^{*}(A)+\epsilon \tilde{h}(A), \tilde{y}^{*}(B)+\epsilon \tilde{h}(B), \tilde{y}^{*}(b)+\epsilon \tilde{h}(b)\right) d x,
\end{gathered}
$$

for $i=1,2$. The lower bound and upper bound of $\tilde{J}$ are

$$
\underline{J}^{r}(\epsilon)=\int_{A}^{B}\left\{\underline{L}^{r}\left[x, \tilde{y}^{*}(x)+\epsilon \tilde{h}(x)\right]_{a, A, B, b}^{r}\right\} d x
$$


and

$$
\bar{J}^{r}(\epsilon)=\int_{A}^{B}\left\{\bar{L}^{r}\left[x, \tilde{y}^{*}(x)+\epsilon \tilde{h}(x)\right]_{a, A, B, b}^{r}\right\} d x
$$

respectively, where

$$
\begin{aligned}
{\left[x, \tilde{y}^{*}(x)+\epsilon \tilde{h}(x)\right.} & ]_{a, A, B, b}^{r} \\
= & \left(x, \underline{y}^{* r}(x)+\epsilon \underline{h}^{r}(x), \bar{y}^{* r}(x)+\epsilon \bar{h}^{r}(x),{ }_{a}^{C} D_{x}^{\alpha}\left(\underline{y}^{* r}+\epsilon \underline{h}^{r}(x)\right),\right. \\
& { }_{a}^{C} D_{x}^{\alpha}\left(\bar{y}^{* r}+\epsilon \bar{h}^{r}(x)\right){ }_{x}^{C} D_{b}^{\beta}\left(\underline{y}^{* r}+\epsilon \underline{h}^{r}(x)\right),{ }_{x}^{C} D_{b}^{\beta}\left(\bar{y}^{* r}+\epsilon \bar{h}^{r}(x)\right), \\
& \underline{y}^{r}(a)+\epsilon \underline{h}^{r}(a), \bar{y}^{* r}(a)+\epsilon \bar{h}(a), \underline{y}^{* r}(A)+\epsilon \underline{h}^{r}(A), \bar{y}^{* r}(A)+\epsilon \bar{h}^{r}(A), \\
& \left.\underline{y}^{r}(B)+\epsilon \underline{h}^{r}(B), \bar{y}^{* r}(B)+\epsilon \bar{h}^{r}(B), \underline{y}^{* r}(b)+\epsilon \underline{h}^{r}(b), \bar{y}^{* r}(b)+\epsilon \bar{h}^{r}(b)\right) .
\end{aligned}
$$

Attending that $\tilde{J}(\epsilon)$ is extremum at $\epsilon=0$, we have the necessary conditions $\frac{d J^{r}}{d \epsilon}=0, \frac{d \bar{J}^{r}}{d \epsilon}=0$, at $\epsilon=0$, for all admissible function $\tilde{h}(x)$. Starting with equation

$$
\left.\frac{d \underline{J}^{r}}{d \epsilon}\right|_{\epsilon=0}=0
$$

we get

$$
\begin{aligned}
\int_{A}^{B} & {\left[\partial_{2} \underline{L}^{r}(\ldots) \underline{h}^{r}+\partial_{3} \underline{L}^{r}(\ldots) \bar{h}^{r}+\partial_{4} \underline{L}^{r}(\ldots)_{a}^{C} D_{x}^{\alpha} \underline{h}^{r}(x)+\partial_{5} \underline{L}^{r}(\ldots)_{a}^{C} D_{x}^{\alpha} \bar{h}^{r}(x)\right.} \\
& +\partial_{6} \underline{L}(\ldots)_{x}^{C} D_{b}^{\beta} \underline{h}^{r}(x)+\partial_{7} \underline{L}^{r}(\ldots)_{x}^{C} D_{b}^{\beta} \bar{h}^{r}(x)+\partial_{8} \underline{L}^{r}(\ldots) \underline{h}^{r}(a) \\
& +\partial_{9} \underline{L}^{r}(\ldots) \bar{h}^{r}(a)+\partial_{10} \underline{L}(\ldots) \underline{h}^{r}(A)+\partial_{11} \underline{L}^{r}(\ldots) \bar{h}^{r}(A) \\
& \left.+\partial_{12} \underline{L}^{r}(\ldots) \underline{h}^{r}(B)+\partial_{13} \underline{L}(\ldots) \bar{h}^{r}(B)+\partial_{14} \underline{L}^{r}(\ldots) \underline{h}^{r}(b)+\partial_{15} \underline{L}^{r}(\ldots) \bar{h}^{r}(b)\right] d x=0,
\end{aligned}
$$


where $(\ldots)=\left[x, \tilde{y}^{*}(x)\right]_{a, A, B, b}^{r}$. Replacing the next four relations

$$
\begin{aligned}
\int_{A}^{B} \partial_{4} \underline{L}^{r}(\ldots)_{a}^{C} D_{x}^{\alpha} \underline{h}^{r}(x) d x= & \int_{a}^{B} \partial_{4} \underline{L}^{r}(\ldots)_{a}^{C} D_{x}^{\alpha} \underline{h}^{r}(x) d x-\int_{a}^{A} \partial_{4} \underline{L}^{r}(\ldots)_{a}^{C} D_{x}^{\alpha} \underline{h}^{r}(x) d x \\
= & \int_{a}^{B} \underline{h}^{r}(x)_{x} D_{B}^{\alpha} \partial_{4} \underline{L}^{r}(\ldots) d x-\int_{a}^{A} \underline{h}^{r}(x)_{x} D_{A}^{\alpha} \partial_{4} \underline{L}^{r}(\ldots) d x \\
& +\left.{ }_{x} I_{B}^{1-\alpha} \partial_{4} \underline{L}^{r}(\ldots) \underline{h}^{r}(x)\right|_{x=a} ^{x=B}-\left.{ }_{x} I_{A}^{1-\alpha} \partial_{4} \underline{L}^{r}(\ldots) \underline{h}^{r}(x)\right|_{x=a} ^{x=A}, \\
\int_{A}^{B} \partial_{5} \underline{L}^{r}\left(\ldots{ }_{a}^{C} D_{x}^{\alpha} \bar{h}^{r}(x) d x=\right. & \int_{a}^{B} \bar{h}^{r}(x)_{x} D_{B}^{\alpha} \partial_{5} \underline{L}^{r}(\ldots) d x-\int_{a}^{A} \bar{h}^{r}(x)_{x} D_{A}^{\alpha} \partial_{5} \underline{L}^{r}(\ldots) d x \\
& +\left.{ }_{x} I_{B}^{1-\alpha} \partial_{5} \underline{L}^{r}(\ldots) \bar{h}^{r}(x)\right|_{x=a} ^{x=B}-\left.{ }_{x} I_{A}^{1-\alpha} \partial_{5} \underline{L}^{r}(\ldots) \bar{h}^{r}(x)\right|_{x=a} ^{x=A}, \\
\int_{A}^{B} \partial_{6} \underline{L}^{r}(\ldots)_{x}^{C} D_{b}^{\beta} \underline{h}^{r}(x) d x= & \int_{A}^{b} \partial_{6} \underline{L}^{r}(\ldots)_{x}^{C} D_{b}^{\beta} \underline{h}^{r}(x) d x-\int_{B}^{b} \partial_{6} \underline{L}^{r}(\ldots)_{x}^{C} D_{b}^{\beta} \underline{h}^{r}(x) d x \\
= & \int_{A}^{b} \underline{h}^{r}(x)_{A} D_{x}^{\beta} \partial_{6} \underline{L}^{r}(\ldots) d x-\int_{B}^{b} \underline{h}^{r}(x)_{B} D_{x}^{\beta} \partial_{6} \underline{L}^{r}(\ldots) d x \\
& -\left.{ }_{A} I_{x}^{1-\beta} \partial_{6} \underline{L}^{r}(\ldots) \underline{h}^{r}(x)\right|_{x=A} ^{x=b}+\left.{ }_{B} I_{x}^{1-\beta} \partial_{6} \underline{L}^{r}(\ldots) \underline{h}^{r}(x)\right|_{x=B} ^{x=b}, \\
\int_{A}^{B} \partial_{7} \underline{L}^{r}\left(\ldots{ }_{x}^{C} D_{b}^{\beta} \bar{h}^{r}(x) d x=\right. & \int_{A}^{b} \bar{h}^{r}(x)_{A} D_{x}^{\beta} \partial_{7} \underline{L}^{r}(\ldots) d x-\int_{B}^{b} \bar{h}^{r}(x)_{B} D_{x}^{\beta} \partial_{7} \underline{L}^{r}(\ldots) d x \\
& -\left.{ }_{A} I_{x}^{1-\beta} \partial_{7} \underline{L}^{r}(\ldots) \bar{h}^{r}(x)\right|_{x=A} ^{x=b}+\left.{ }_{B} I_{x}^{1-\beta} \partial_{7} \underline{L}^{r}(\ldots) \bar{h}^{r}(x)\right|_{x=B} ^{x=b},
\end{aligned}
$$

into Eq. (17), doing the same computations as presented before, and by the arbitrariness of $\tilde{h}$, we obtain the following result.

Theorem 3.5. Let $\tilde{y}^{*}$ be a local extremizer to problem (16). Then, $\tilde{y}$ satisfies the fractional Euler-Lagrange equations

$$
\left\{\begin{array}{l}
{ }_{x} D_{B}^{\alpha} \partial_{4} \underline{L}^{r}(\ldots)-{ }_{x} D_{A}^{\alpha} \partial_{4} \underline{L}^{r}(\ldots)=0 \\
{ }_{x} D_{B}^{\alpha} \partial_{4} \bar{L}^{r}(\ldots)-{ }_{x} D_{A}^{\alpha} \partial_{4} \bar{L}^{r}(\ldots)=0 \\
{ }_{x} D_{B}^{\alpha} \partial_{5} \underline{L}^{r}(\ldots)-{ }_{x} D_{A}^{\alpha} \partial_{5} \underline{L}^{r}(\ldots)=0 \\
{ }_{x} D_{B}^{\alpha} \partial_{5} \bar{L}^{r}(\ldots)-{ }_{x} D_{A}^{\alpha} \partial_{5} \bar{L}^{r}(\ldots)=0
\end{array}\right.
$$

for all $x \in[a, A]$,

$$
\left\{\begin{array}{l}
\partial_{2} \underline{L}^{r}(\ldots)+{ }_{x} D_{B}^{\alpha} \partial_{4} \underline{L}^{r}(\ldots)+{ }_{A} D_{x}^{\beta} \partial_{6} \underline{L}^{r}(\ldots)=0 \\
\partial_{2} \bar{L}^{r}(\ldots)+{ }_{x} D_{B}^{\alpha} \partial_{4} \bar{L}^{r}(\ldots)+{ }_{A} D_{x}^{\beta} \partial_{6} \bar{L}^{r}(\ldots)=0 \\
\partial_{3} \underline{L}^{r}(\ldots)+{ }_{x} D_{B}^{\alpha} \partial_{5} \underline{L}^{r}(\ldots)+{ }_{A} D_{x}^{\beta} \partial_{7} \underline{L}^{r}(\ldots)=0 \\
\partial_{3} \bar{L}^{r}(\ldots)+{ }_{x} D_{B}^{\alpha} \partial_{5} \bar{L}^{r}(\ldots)+{ }_{A} D_{x}^{\beta} \partial_{7} \bar{L}^{r}(\ldots)=0
\end{array}\right.
$$

for all $x \in[A, B]$,

$$
\left\{\begin{array}{l}
{ }_{A} D_{x}^{\beta} \partial_{6} \underline{L}^{r}(\ldots)-{ }_{B} D_{x}^{\beta} \partial_{6} \underline{L}^{r}(\ldots)=0 \\
{ }_{A} D_{x}^{\beta} \partial_{6} \bar{L}^{r}(\ldots)-{ }_{B} D_{x}^{\beta} \partial_{6} \bar{L}^{r}(\ldots)=0 \\
{ }_{A} D_{x}^{\beta} \partial_{7} \underline{L}^{r}(\ldots)-{ }_{B} D_{x}^{\beta} \partial_{7} \underline{L}^{r}(\ldots)=0 \\
{ }_{A} D_{x}^{\beta} \partial_{7} \bar{L}^{r}(\ldots)-{ }_{B} D_{x}^{\beta} \partial_{7} \bar{L}^{r}(\ldots)=0
\end{array}\right.
$$


for all $x \in[B, b]$. Moreover, if $\tilde{y}(a)$ is free, then

$$
\left\{\begin{array}{l}
\int_{A}^{B} \partial_{8} \underline{L}^{r}(\ldots) d x-\left.\left({ }_{x} I_{B}^{1-\alpha} \partial_{4} \underline{L}^{r}(\ldots)-{ }_{x} I_{A}^{1-\alpha} \partial_{4} \underline{L}^{r}(\ldots)\right)\right|_{x=a}=0 \\
\int_{A}^{B} \partial_{8} \bar{L}^{r}(\ldots) d x-\left.\left({ }_{x} I_{B}^{1-\alpha} \partial_{4} \bar{L}^{r}(\ldots)-{ }_{x} I_{A}^{1-\alpha} \partial_{4} \bar{L}^{r}(\ldots)\right)\right|_{x=a}=0 \\
\int_{A}^{B} \partial_{9} \underline{L}^{r}(\ldots) d x-\left.\left({ }_{x} I_{B}^{1-\alpha} \partial_{5} \underline{L}^{r}(\ldots)-{ }_{x} I_{A}^{1-\alpha} \partial_{5} \underline{L}^{r}(\ldots)\right)\right|_{x=a}=0 \\
\int_{A}^{B} \partial_{9} \bar{L}^{r}(\ldots) d x-\left.\left({ }_{x} I_{B}^{1-\alpha} \partial_{5} \bar{L}^{r}(\ldots)-{ }_{x} I_{A}^{1-\alpha} \partial_{5} \bar{L}^{r}(\ldots)\right)\right|_{x=a}=0
\end{array}\right.
$$

when $\tilde{y}(A)$ is free, then

$$
\left\{\begin{array}{l}
\int_{A}^{B} \partial_{10} \underline{L}^{r}(\ldots) d x-\left.\left({ }_{x} I_{A}^{1-\alpha} \partial_{4} \underline{L}^{r}(\ldots)-{ }_{A} I_{x}^{1-\beta} \partial_{6} \underline{L}^{r}(\ldots)\right)\right|_{x=A}=0 \\
\int_{A}^{B} \partial_{10} \bar{L}^{r}(\ldots) d x-\left.\left({ }_{x} I_{A}^{1-\alpha} \partial_{4} \bar{L}^{r}(\ldots)-{ }_{A} I_{x}^{1-\beta} \partial_{6} \bar{L}^{r}(\ldots)\right)\right|_{x=A}=0 \\
\int_{A}^{B} \partial_{11} \underline{L}^{r}(\ldots) d x-\left.\left({ }_{x} I_{A}^{1-\alpha} \partial_{5} \underline{L}^{r}(\ldots)-{ }_{A} I_{x}^{1-\beta} \partial_{7} \underline{L}^{r}(\ldots)\right)\right|_{x=A}=0 \\
\int_{A}^{B} \partial_{11} \bar{L}^{r}(\ldots) d x-\left.\left({ }_{x} I_{A}^{1-\alpha} \partial_{5} \bar{L}^{r}(\ldots)-{ }_{A} I_{x}^{1-\beta} \partial_{7} \bar{L}^{r}(\ldots)\right)\right|_{x=A}=0
\end{array}\right.
$$

when $\tilde{y}(B)$ is free, then

$$
\left\{\begin{array}{l}
\int_{A}^{B} \partial_{12} \underline{L}^{r}(\ldots) d x-\left.\left({ }_{B} I_{x}^{1-\beta} \partial_{6} \underline{L}^{r}(\ldots)-{ }_{x} I_{B}^{1-\alpha} \partial_{4} \underline{L}^{r}(\ldots)\right)\right|_{x=B}=0, \\
\int_{A}^{B} \partial_{12} \bar{L}^{r}(\ldots) d x-\left.\left({ }_{B} I_{x}^{1-\beta} \partial_{6} \bar{L}^{r}(\ldots)-{ }_{x} I_{B}^{1-\alpha} \partial_{4} \bar{L}^{r}(\ldots)\right)\right|_{x=B}=0, \\
\int_{A}^{B} \partial_{13} \underline{L}^{r}(\ldots) d x-\left.\left({ }_{B} I_{x}^{1-\beta} \partial_{7} \underline{L}^{r}(\ldots)-{ }_{x} I_{B}^{1-\alpha} \partial_{5} \underline{L}^{r}(\ldots)\right)\right|_{x=B}=0, \\
\int_{A}^{B} \partial_{13} \bar{L}^{r}(\ldots) d x-\left.\left({ }_{B} I_{x}^{1-\beta} \partial_{7} \bar{L}^{r}(\ldots)-{ }_{x} I_{B}^{1-\alpha} \partial_{5} \bar{L}^{r}(\ldots)\right)\right|_{x=B}=0 ;
\end{array}\right.
$$

and when $\tilde{y}(b)$ is free, then

$$
\left\{\begin{array}{l}
\int_{A}^{B} \partial_{14} \underline{L}^{r}(\ldots) d x-\left({ }_{A} I_{x}^{1-\beta} \partial_{6} \underline{L}^{r}(\ldots)-\left.{ }_{B} I_{x}^{1-\beta} \partial_{6} \underline{L}^{r}(\ldots)\right|_{x=b}=0,\right. \\
\int_{A}^{B} \partial_{14} \bar{L}^{r}(\ldots) d x-\left({ }_{A} I_{x}^{1-\beta} \partial_{6} \bar{L}^{r}(\ldots)-\left.{ }_{B} I_{x}^{1-\beta} \partial_{6} \bar{L}^{r}(\ldots)\right|_{x=b}=0,\right. \\
\int_{A}^{B} \partial_{15} \underline{L}^{r}(\ldots) d x-\left({ }_{A} I_{x}^{1-\beta} \partial_{7} \underline{L}^{r}(\ldots)-\left.{ }_{B} I_{x}^{1-\beta} \partial_{7} \underline{L}^{r}(\ldots)\right|_{x=b}=0,\right. \\
\int_{A}^{B} \partial_{15} \bar{L}^{r}(\ldots) d x-\left.\left({ }_{A} I_{x}^{1-\beta} \partial_{7} \bar{L}^{r}(\ldots)-{ }_{B} I_{x}^{1-\beta} \partial_{7} \bar{L}^{r}(\ldots)\right)\right|_{x=b}=0,
\end{array}\right.
$$

where

$$
\begin{aligned}
(\ldots)= & \left(x, \underline{y}^{* r}(x), \bar{y}^{* r}(x),{ }_{a}^{C} D_{x}^{\alpha} \underline{y}^{* r},{ }_{a}^{C} D_{x}^{\alpha} \bar{y}^{* r},{ }_{x}^{C} D_{b}^{\beta} \underline{y}^{* r},{ }_{x}^{C} D_{b}^{\beta} \bar{y}^{* r},\right. \\
& \left.\underline{y}^{* r}(a), \bar{y}^{* r}(a), \underline{y}^{* r}(A), \bar{y}^{* r}(A), \underline{y}^{* r}(B), \bar{y}^{* r}(B), \underline{y}^{* r}(b), \bar{y}^{* r}(b)\right) .
\end{aligned}
$$

\section{Transversality for Fuzzy Fractional Variational Problems}

In this section, for simplicity and without lose of generality, we consider the following FFVP described by

$$
\begin{gathered}
\tilde{J}(\tilde{y})=\int_{a}^{b} \tilde{L}\left(x, \tilde{y}(x),{ }^{g H-C}{ }_{a} \mathcal{D}_{i x}^{\alpha} \tilde{y}(x)\right) d x \longrightarrow \text { extr, } \\
\tilde{y}(a)=\tilde{y}_{a}
\end{gathered}
$$

and $\tilde{y}(x)$ intersects the curve $\tilde{z}=\tilde{C}(x)$ for the first time at $b$, i.e. $\tilde{y}(b)=\tilde{C}(b)$. Here, $\tilde{C}(x)$ is a specified curve. We implicitly assume that all differentiability conditions are met. Note that in this problem we do not know the end point $x=b$ in advance except that the end point of $\tilde{y}(x)$ lies on a specified curve.

To develop the necessary conditions for this problem, assume that $\tilde{y}^{*}(x)$ is the desired function which intersects the curve $\tilde{z}=\tilde{C}(x)$ at $x=b^{*}$, i.e. $\tilde{y}^{*}\left(b^{*}\right)=\tilde{C}\left(b^{*}\right)$. Take $\epsilon \in \mathbb{R}$ and define a family of curves

$$
\tilde{y}(x)=\tilde{y}^{*}(x)+\epsilon \tilde{h}(x)
$$


where $\tilde{h}(x)$ is an arbitrary fuzzy function which satisfy the boundary conditions, i.e., we require that $\tilde{h}(a)=\tilde{0}$. The lower bound and upper bound of $\tilde{y}$ are

$$
\underline{y}^{r}(x)=\underline{y}^{r *}(x)+\epsilon \underline{h}^{r}(x) \text { and } \bar{y}^{r}(x)=\bar{y}^{r *}(x)+\epsilon \bar{h}^{r}(x) .
$$

We further define a set of end-points

$$
b=b^{*}+\epsilon \zeta\left(b^{*}\right)
$$

where $\zeta\left(b^{*}\right)$ is a variation in $b^{*}$.

The lower bound and upper bound of $\tilde{J}$ are

$$
\begin{aligned}
& \underline{J}^{r}(\epsilon)=\int_{a}^{b} \underline{L}^{r}\left(x, \underline{y}^{r}(x), \bar{y}^{r}(x),{ }_{a}^{C} D_{x}^{\alpha} \underline{y}^{r}(x),{ }_{a}^{C} D_{x}^{\alpha} \bar{y}^{r}(x)\right) d x, \\
& \bar{J}^{r}(\epsilon)=\int_{a}^{b} \bar{L}^{r}\left(x, \underline{y}^{r}(x), \bar{y}^{r}(x),{ }_{a}^{C} D_{x}^{\alpha} \underline{y}^{r}(x),{ }_{a}^{C} D_{x}^{\alpha} \bar{y}^{r}(x)\right) d x .
\end{aligned}
$$

To obtain the extremum of the functional, we substitute equations (20) and (21) into equations (22) and (23), find the expression for $\frac{d J^{r}}{d \epsilon}$ and $\frac{d \bar{J}^{r}}{d \epsilon}$, and set $\epsilon, \frac{d J^{r}}{d \epsilon}$ and $\frac{d \bar{J}^{r}}{d \epsilon}$ equal to 0 . This leads to

$$
\begin{array}{r}
\int_{a}^{b^{*}}\left\{\partial_{2} \underline{L}^{r}(\ldots) \underline{h}^{r}+\partial_{3} \underline{L}^{r}(\ldots) \bar{h}^{r}+\partial_{4} \underline{L}^{r}(\ldots){ }_{a}^{C} D_{x}^{\alpha} \underline{h}^{r}+\partial_{5} \underline{L}^{r}(\ldots){ }_{a}^{C} D_{x}^{\alpha} \bar{h}^{r}\right\} d x \\
+\zeta\left(b^{*}\right)\left[\underline{L}^{r}\left(b^{*}, \underline{y}^{r *}\left(b^{*}\right), \bar{y}^{r *}\left(b^{*}\right),{ }_{a}^{C} D_{x}^{\alpha} \underline{y}^{r *}\left(b^{*}\right),{ }_{a}^{C} D_{x}^{\alpha} \bar{y}^{r *}\left(b^{*}\right)\right)\right]=0
\end{array}
$$

and

$$
\begin{array}{r}
\int_{a}^{b^{*}}\left\{\partial_{2} \bar{L}^{r}(\ldots) \underline{h}^{r}+\partial_{3} \bar{L}^{r}(\ldots) \bar{h}^{r}+\partial_{4} \bar{L}^{r}(\ldots){ }_{a}^{C} D_{x}^{\alpha} \underline{h}^{r}+\partial_{5} \bar{L}^{r}(\ldots){ }_{a}^{C} D_{x}^{\alpha} \bar{h}^{r}\right\} d x \\
+\zeta\left(b^{*}\right)\left[\bar{L}^{r}\left(b^{*}, \underline{y}^{r *}\left(b^{*}\right), \bar{y}^{r *}\left(b^{*}\right){ }_{a}^{C} D_{x}^{\alpha} \underline{y}^{r *}\left(b^{*}\right){ }_{a}^{C} D_{x}^{\alpha} \bar{y}^{r *}\left(b^{*}\right)\right)\right]=0,
\end{array}
$$

where

$$
(\ldots)=\left(x, \underline{y}^{* r}(x), \bar{y}^{* r}(x){ }_{a}^{C} D_{x}^{\alpha} \underline{y}^{* r}{ }_{a}^{C} D_{x}^{\alpha} \bar{y}^{* r}\right) .
$$

Note that $b^{*}$ is still unknown. However, it is a fixed point, therefore it can be used to define the right fractional derivative.

Using integration by parts and terminal condition $\tilde{y}(a)=\tilde{y}_{a}$, equation (24) can be written as

$$
\begin{aligned}
\int_{a}^{b^{*}} & \left\{\left[\partial_{2} \underline{L}^{r}(\ldots)+{ }_{x} D_{b^{*}}^{\alpha} \partial_{4} \underline{L}^{r}(\ldots)\right] \underline{h}^{r}+\left[\partial_{3} \underline{L}^{r}(\ldots)+{ }_{x} D_{b^{*}}^{\alpha} \partial_{5} \underline{L}^{r}(\ldots)\right] \bar{h}^{r}\right\} d x \\
& +\left.\left[{ }_{x} I_{b^{*}}^{1-\alpha} \partial_{4} \underline{L}^{r}(\ldots) \underline{h}^{r}(x)+{ }_{x} I_{b^{*}}^{1-\alpha} \partial_{5} \underline{L}^{r}(\ldots) \bar{h}^{r}(x)\right]\right|_{x=b^{*}} \\
& +\zeta\left(b^{*}\right) \cdot\left(\underline{L}^{r}\left(b^{*}, \underline{y}^{r *}\left(b^{*}\right), \bar{y}^{r *}\left(b^{*}\right),{ }_{a}^{C} D_{x}^{\alpha} \underline{y}^{r *}\left(b^{*}\right),{ }_{a}^{C} D_{x}^{\alpha} \bar{y}^{r *}\left(b^{*}\right)\right)\right)=0 .
\end{aligned}
$$

Since the right end point lies on the curve $\tilde{z}=\tilde{C}(x)$, we have from equations (19) and (21),

$$
\tilde{y}^{*}\left(b^{*}+\epsilon \zeta\left(b^{*}\right)\right)+\epsilon \tilde{h}\left(b^{*}+\epsilon \zeta\left(b^{*}\right)\right)=\tilde{C}\left(b^{*}+\epsilon \zeta\left(b^{*}\right)\right)
$$

with $r$-level set $\left[\underline{y}^{r *}, \bar{y}^{r *}\right]$ where

$$
\begin{aligned}
& \underline{y}^{r *}\left(b^{*}+\epsilon \zeta\left(b^{*}\right)\right)+\epsilon \underline{h}^{r}\left(b^{*}+\epsilon \zeta\left(b^{*}\right)\right)=\underline{C}^{r}\left(b^{*}+\epsilon \zeta\left(b^{*}\right)\right), \\
& \bar{y}^{r *}\left(b^{*}+\epsilon \zeta\left(b^{*}\right)\right)+\epsilon \bar{h}^{r}\left(b^{*}+\epsilon \zeta\left(b^{*}\right)\right)=\bar{C}^{r}\left(b^{*}+\epsilon \zeta\left(b^{*}\right)\right) .
\end{aligned}
$$


Differentiating equations (28) and (29) with respect to $\epsilon$ and then setting $\epsilon=0$, we obtain

$$
\begin{aligned}
& \underline{h}^{r}\left(b^{*}\right)=\zeta\left(b^{*}\right) \cdot\left(D \underline{C}^{r}\left(b^{*}\right)-D \underline{y}^{r *}\left(b^{*}\right)\right), \\
& \bar{h}^{r}\left(b^{*}\right)=\zeta\left(b^{*}\right) \cdot\left(D \bar{C}^{r}\left(b^{*}\right)-D \bar{y}^{r *}\left(b^{*}\right)\right) .
\end{aligned}
$$

Using equations (26), (30) and (31), the generalized Euler-Lagrange equations and the transversality conditions can be written, respectively, as

$$
\begin{aligned}
& \partial_{2} \underline{L}^{r}(\ldots)+{ }_{x} D_{b^{*}}^{\alpha} \partial_{4} \underline{L}^{r}(\ldots)=0, \\
& \partial_{3} \underline{L}^{r}(\ldots)+{ }_{x} D_{b^{*}}^{\alpha} \partial_{5} \underline{L}^{r}(\ldots)=0,
\end{aligned}
$$

for all $x \in\left[a, b^{*}\right]$, and

$$
\begin{aligned}
\zeta\left(b^{*}\right) \cdot\left[\left({ }_{x} I_{b^{*}}^{1-\alpha} \partial_{4} \underline{L}^{r}(\ldots)\right) \cdot\left(D \underline{C}^{r}-D \underline{y}^{r *}\right)\right. \\
\left.\quad+\left({ }_{x} I_{b^{*}}^{1-\alpha} \partial_{5} \underline{L}^{r}(\ldots)\right)\left(D \bar{C}^{r}-D \bar{y}^{r *}\right)+\underline{L}^{r}\right]=0 \text {, for } x=b^{*} .
\end{aligned}
$$

In general $\zeta\left(b^{*}\right)$ is not 0 , therefore the coefficient of $\zeta\left(b^{*}\right)$ in above equation must be 0 . This gives the following transversality condition for the problem

$$
\begin{aligned}
\left({ }_{x} I_{b^{*}}^{1-\alpha} \partial_{4} \underline{L}^{r}(\ldots)\right) & \cdot\left(D \underline{C}^{r}-D \underline{y}^{r *}\right) \\
& +\left({ }_{x} I_{b^{*}}^{1-\alpha} \partial_{5} \underline{L}^{r}(\ldots)\right) \cdot\left(D \bar{C}^{r}-D \bar{y}^{r *}\right)+\underline{L}^{r}=0, \text { for } x=b^{*} .
\end{aligned}
$$

Following the scheme of obtaining (32)-(34) and adapting it to the case under consideration involving (25), one can show that

$$
\begin{gathered}
\partial_{2} \bar{L}^{r}(\ldots)+{ }_{x} D_{b^{*}}^{\alpha} \partial_{4} \bar{L}^{r}(\ldots)=0, \\
\partial_{3} \bar{L}^{r}(\ldots)+{ }_{x} D_{b^{*}}^{\alpha} \partial_{5} \bar{L}^{r}(\ldots)=0, \\
\left({ }_{x} I_{b^{*}}^{1-\alpha} \partial_{4} \bar{L}^{r}(\ldots)\right) \cdot\left(D \underline{C}^{r}-D \underline{y}^{r *}\right) \\
+\left({ }_{x} I_{b^{*}}^{1-\alpha} \partial_{5} \bar{L}^{r}(\ldots)\right) \cdot\left(D \bar{C}^{r}-D \bar{y}^{r *}\right)+\bar{L}^{r}=0, \text { for } x=b^{*} .
\end{gathered}
$$

Eqs.(34) and (37) are called transversality conditions.

Example 4.1. Consider the following fuzzy fractional variational problem:

$$
\begin{gathered}
\tilde{J}(\tilde{y}):=\int_{1}^{b}\left({ }^{g H-C}{ }_{1} \mathcal{D}_{i x}^{\alpha} \tilde{y}\right)^{2} x^{3} d x \longrightarrow \min , \\
\tilde{y}(1)=<-1,0,1>
\end{gathered}
$$

where $b$ is finite and $\tilde{y}(b)$ lies on the curve $\tilde{y}=\frac{\tilde{2}}{x^{2}}-\tilde{3}$, given that $\tilde{2}=<1,2,3>$ and $\tilde{3}=<2,3,4>$.

Solution. Suppose that $\tilde{y}(x)$ is $[(1)-g H]$-differentiable function. The $r$-level set of $\tilde{J}$ is:

$$
[\tilde{J}(\tilde{y})]^{r}=\left[\int_{1}^{b}\left({ }_{1}^{C} D_{x}^{\alpha} \underline{y}^{r}\right)^{2} x^{3} d x, \int_{1}^{b}\left({ }_{1}^{C} D_{x}^{\alpha} \bar{y}^{r}\right)^{2} x^{3} d x\right] .
$$

The objective curve is $\tilde{C}(x)=\frac{\tilde{2}}{x^{2}}-\tilde{3}$ where $\underline{C}^{r}=\frac{r+1}{x^{2}}-4+r$ and $\bar{C}^{r}=\frac{3-r}{x^{2}}-4-r$, so we have $D \underline{C}^{r}=\frac{-2(r+1)}{x^{3}}$, and $D \bar{C}^{r}=\frac{-2(3-r)}{x^{3}}$. 
From fuzzy Euler-Lagrange equations and transversality conditions in (32)-(37) we have

$$
\begin{gathered}
\left.{ }_{x} D_{b}^{\alpha}\left(2{ }_{1}^{C} D_{x}^{\alpha} \underline{y}^{r}\right) x^{3}\right)=0, \\
\left.{ }_{x} D_{b}^{\alpha}\left(2{ }_{1}^{C} D_{x}^{\alpha} \bar{y}^{r}\right) x^{3}\right)=0, \\
\left({ }_{x} I_{b}^{1-\alpha}\left(2{ }_{1}^{C} D_{x}^{\alpha} \underline{y}^{r}\right) x^{3}\right) \cdot\left(\frac{-2(r+1)}{x^{3}}-D \underline{y}^{r}\right)+\left({ }_{1}^{C} D_{x}^{\alpha} \underline{y}^{r}\right)^{2} \cdot x^{3}=0, \text { for } x=b^{*}, \\
\left({ }_{x} I_{b}^{1-\alpha}\left(2{ }_{1}^{C} D_{x}^{\alpha} \bar{y}^{r}\right) x^{3}\right) \cdot\left(\frac{-2(r+1)}{x^{3}}-D \bar{y}^{r}\right)+\left({ }_{1}^{C} D_{x}^{\alpha} \bar{y}^{r}\right)^{2} x^{3}=0, \text { for } x=b^{*} .
\end{gathered}
$$

Eqs. (38) must be solved to find the solution to the problem. If we set $\alpha=1$, then

$$
\begin{aligned}
& -\frac{d}{d x}\left(2 \dot{y}^{r} x^{3}\right)=0, \\
& -\frac{d}{d x}\left(2 \dot{\bar{y}}^{r} x^{3}\right)=0 .
\end{aligned}
$$

By virtue of the classical differential equation theory, we may solve it analytically for fixed $r \in[0,1]$ to arrive at

$$
\underline{y}^{r}=\frac{k_{1}}{x^{2}}+r-1-k_{1}, \bar{y}^{r}=\frac{k_{2}}{x^{2}}+1-r-k_{2},
$$

so

$$
\underline{\dot{y}}^{r}=-\frac{2 k_{1}}{x^{3}}, \dot{\bar{y}}^{r}=-\frac{2 k_{2}}{x^{3}} .
$$

From transversality conditions (34) and (37)

$$
\begin{aligned}
& -\frac{4 k_{1}}{b^{3}} b^{3}\left(-\frac{2(r+1)}{b^{3}}+\frac{2 k_{1}}{b^{3}}\right)+\left(-\frac{2 k_{1}}{b^{3}}\right)^{2} \cdot b^{3}=0, \\
& -\frac{4 k_{2}}{b^{3}} b^{3}\left(-\frac{2(3-r)}{b^{3}}+\frac{2 k_{2}}{b^{3}}\right)+\left(-\frac{2 k_{2}}{b^{3}}\right)^{2} \cdot b^{3}=0 .
\end{aligned}
$$

Since $b$ is finite we have $k_{1}=2(r+1)$ and $k_{2}=2(3-r)$. Hence, we arrive at the extremum $\tilde{y}(x)$ where

$$
[\tilde{y}(x)]^{r}=\left[\frac{2(r+1)}{x^{2}}-r-3, \frac{2(3-r)}{x^{2}}+r-5\right]
$$

and intersects $\tilde{y}=\frac{\tilde{2}}{x^{2}}-\tilde{3}$ at $b=\sqrt{2}$. This solution is shown in Figure 2 .

\section{Conclusion}

In this paper we considered a new class of fuzzy fractional functionals of the calculus of variations containing fuzzy fractional derivatives Liouville-Caputo senses. We provided necessary optimality conditions for such variational functionals. The Liouville-Caputo derivative demands higher conditions of regularity for differentiability: to compute the fractional derivative of a function in the Liouville-Caputo sense, we must first calculate its derivative. Liouville-Caputo derivatives are defined only for differentiable functions while functions that have no first order derivative might have fractional derivatives of all orders less than one in the Riemann-Liouville sense. In a future paper, we aim to investigate this class of problems in the sense of fuzzy Riemann-Liouville derivative. 


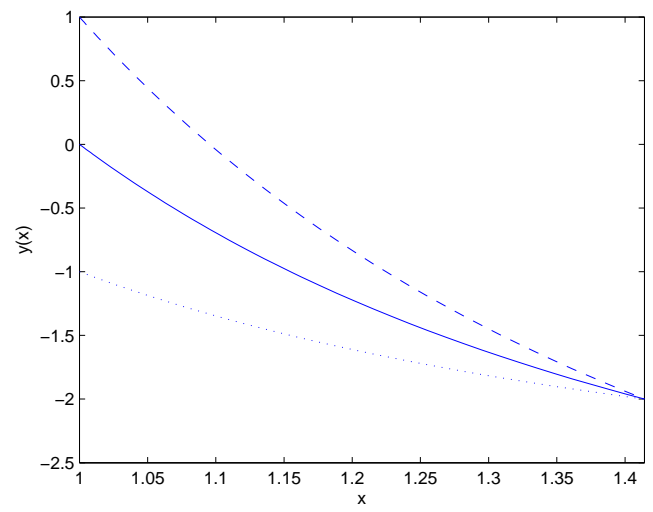

FiguRE 2. solution of Example 4.1

$\underline{y}^{0}(x)$ (dotted line), $\bar{y}^{0}(x)$ (dashed line), $\underline{y}^{1}(x)=\bar{y}^{1}(x)$ (solid line).

\section{ACKNowledgments}

R. Almeida was supported by Portuguese funds through the CIDMA - Center for Research and Development in Mathematics and Applications, and the Portuguese Foundation for Science and Technology (FCT-Fundação para a Ciência e a Tecnologia), within project PEst-OE/MAT/UI4106/2014.

\section{REFERENCES}

[1] O. P. Agrawal, Formulation of Euler-Lagrange equations for fractional variational problems, J. Math. Anal. Appl. 272 (2002) 368-379.

[2] O. P. Agrawal, Fractional variational calculus and the transversality conditions, J. Phys. A 39 (33) (2006) 10375-10384.

[3] O. P. Agrawal, Generalized Euler-Lagrange equations and transversality conditions for FVPs in terms of the Caputo derivative, J. Vib. Control 13 (9-10) (2007) 1217-1237.

[4] T. Allahviranloo, Z. Gouyandeh and A. Armand, Fuzzy fractional differential equations under generalized fuzzy Caputo derivative, Journal of Intelligent Fuzzy Systems 26 (2014) 1481-90.

[5] R. Almeida and D. F. M. Torres, Necessary and sufficient conditions for the fractional calculus of variations with Caputo derivatives, Commun. Nonlinear Sci. Numer. Simul. 16 (3) (2011) $1490-1500$.

[6] R. Almeida, S. Pooseh and D. F. M. Torres, Computational methods in the fractional calculus of variations, Imp. Coll. Press, London; 2015.

[7] S. Arshad and V. Lupulescu, On the fractional differential equations with uncertainty, Nonlinear Anal 74 (2011) 3685-93.

[8] B. Bede and L. Stefanini, Generalized differentiability of fuzzy-valued functions, Fuzzy Sets and Systems 230 (2013) 119-41.

[9] J. J. Buckley and T. Feuring, Introduction to fuzzy partial differential equations, Fuzzy Set. Syst. 105 (1999) 241-248.

[10] R. Caponetto, G. Dongola, L. Fortuna and I. Petras, Fractional order systems: modeling and control applications, Singapore: World Scientific; 2010.

[11] S. Das, Functional fractional calculus for system identification and controls, Berlin Heidelberg: Springer; 2008.

[12] O. S. Fard and M. S. Zadeh, Note on "Necessary optimality conditions for fuzzy variational problems", J. Adv. Res. Dyn. Control Syst. 4(3) (2012) 1-9.

[13] O. S. Fard, A. H. Borzabadi and M. Heidari, On fuzzy Euler-Lagrange equations, Annals of Fuzzy Mathematics and Informatics 7 (3) (2014), 447-461. 
[14] O. S. Fard and M. Salehi, A survey on fuzzy fractional variational problems, Journal of Computational and Applied Mathematics 271 (2014) 71-82.

[15] B. Farhadinia, Necessary optimality conditions for fuzzy variational problems, Information Sciences 181 (2011) 1348-1357

[16] R. Goetschel and W. Voxman, Elementary fuzzy calculus, Fuzzy Sets and Systems 18 (1) (1986) 31-43.

[17] R. Hilfer, Applications of fractional calculus in physics, Singapore: World Scientific Pub Co; 2000.

[18] N. V. Hoa, Fuzzy fractional functional differential equations under Caputo gHdifferentiability, Communications in Nonlinear Science and Numerical Simulation (2014), doi: http://dx.doi.org/10.1016/j.cnsns.2014.08.006

[19] A. A. Kilbas, H. M. Srivastava and J. J. Trujillo, Theory and applications of fractional differential equations, North-Holland Mathematics Studies, 204, Elsevier, Amsterdam, 2006.

[20] A. B. Malinowska and D. F. M. Torres, Generalized natural boundary conditions for fractional variational problems in terms of the Caputo derivative, Computers and Mathematics with Applications 59 (2010) 3110-3116.

[21] A. B. Malinowska and D. F. M. Torres, Multiobjective fractional variational calculus in terms of a combined Caputo derivative, Applied Mathematics and Computation 218 (2012) 5099-5111.

[22] A. B. Malinowska and D. F. M. Torres, Natural boundary conditions in the calculus of variations, Math. Methods Appl. Sci. 33 (14) (2010) 1712-1722.

[23] A. B. Malinowska and D. F. M. Torres, Introduction to the fractional calculus of variations, Imp. Coll. Press, London; 2012.

[24] T. Odzijewicz, A. B. Malinowska and D. F. M. Torres, Advanced methods in the fractional calculus of variations, Springer Briefs in Applied Sciences and Technology, Springer; 2015.

[25] I. Podlubny, Fractional Differential Equations, Academic Press, New York; 1999.

[26] F. Riewe, Nonconservative Lagrangian and Hamiltonian mechanics, Phys. Rev. E 53 (1996) 1890-1899.

[27] S. Salahshour, T. Allahviranloo, S. Abbasbandy and D. Baleanu, Existence and uniqueness results for fractional differential equations with uncertainty, Advances in Difference Equations 2012, 112 (2012).

[28] B. van Brunt, The Calculus of Variations, Springer-Verlag, Heidelberg; 2004.

[29] J. Xu, Z. Liao and J. J. Nieto, A class of linear differential dynamical systems with fuzzy matrices, J. Math. Anal. Appl. 368 (1) (2010) 54-68.

OMid SOLAYMANi FARD

School of Mathematics and Computer Science, Damghan University, Damghan, Iran

E-mail address: osfard@du.ac.ir, omidsfard@gmail.com

Ricardo Almeida

Center for Research and Development in Mathematics and Applications (Cidma), Department of Mathematics, University of Aveiro, 3810-193 Aveiro, Portugal

E-mail address: ricardo.almeida@ua.pt

JAVAD SOOLAKI

Department of Mathematics, Damghan University, Damghan, Iran

E-mail address: javad.soolaki@gmail.com

Akbar Hashemi Borzabadi

School of Mathematics and Computer Science, Damghan University, Damghan, Iran

E-mail address: borzabadi@du.ac.ir 